How to reference this article Pifko-Wadowska, A. (2021). Michał Wiszniewski (1794-1865), italianista, studioso e traduttore di Dante. Italica Wratislaviensia, 12(2), 169-185.

DOI: http://dx.doi.org/10.15804/IW.2021.12.2.09

Anna Pifko-Wadowska

Uniwersytet Jagielloński w Krakowie, Polonia

anna.pifko@student.uj.edu.pl

ORCID: 0000-0002-3214-3474

\title{
MICHAŁ WISZNIEWSKI (1794-1865), \\ ITALIANISTA, STUDIOSO \\ E TRADUTTORE DI DANTE*
}

\author{
MICHAL WISZNIEWSKI (1794-1865), \\ ITALIANIST, DANTE SCHOLAR, AND TRANSLATOR
}

\begin{abstract}
This study focuses on Michał Wiszniewski's interest in Dante. Wiszniewski was a professor of history and literature at the Jagiellonian University of Krakow and was fond of Italy. This work aims to outline Wiszniewski's contribution to the Polish reception of Dante in the first decades of the $19^{\text {th }}$ century. The study recalls Wiszniewski's three Italian trips and his book, Podróż do Włoch, Sycylii i Malty (A Journey to Italy, Sicily and Malta), which contains numerous mentions of Alighieri. The main part of the study is dedicated to the analysis of his manuscript Dante Alighieri, kept in the Jagiellonian Library (ms. 948 fasc. 7), which has not been carefully examined until now. This manuscript features an outline of a portion of a literature course taught by Wiszniewski in the 1830s, a lesson concerning the life and work of Dante (particularly the Commedia). The professor describes the two faces of Dante: the scholastic one (in prose) and the poetic one (in verse); explains the poem's title and the structure of the afterlife; admires the extraordinary plasticity of Dante's images; and translates into Polish a passage from the Convivio and three episodes from the Inferno. The content of his lesson is compared with other earlier and contemporary Polish studies (of very limited number) on the Poet, as well as with the general trends of Dante criticism of that time and with the Dante scholarship of the Polish Romantic poets. The analysis carried out shows that Wiszniewski's contribution to Dante studies is, in principle, close to Romantic criticism, but it also follows some Neoclassical trends. In the present study, we do not analyse the article Studia nad Dantem (1847) because of its uncertain attribution (as we try to show).
\end{abstract}

Keywords: Wiszniewski, Dante, reception, Poland, $19^{\text {th }}$ century

* Studio finanziato con i fondi per la ricerca degli anni 2015-2022 in quanto progetto realizzato nell'ambito del programma "Diamentowy Grant" del Ministero dell'Educazione e della Scienza. 
$\mathrm{La}$ storia della ricezione di Dante in Polonia, pur essendo stata sante campo da indagare. Lo studio dei materiali manoscritti, innanzitutto ottocenteschi, custoditi nelle biblioteche polacche (e non solo) può colmare delle lacune, evidenziando al contempo, ancora di più, il livello della conoscenza dell'Alighieri nella Polonia del XIX secolo' ${ }^{1}$. Tra i materiali inediti e al giorno d'oggi quasi dimenticati si trova il contributo dantesco di Michał Wiszniewski (1794-1865), professore dell'Università Jagellonica, docente di storia e letteratura, uno dei più grandi intellettuali cracoviani dell'epoca, oggi noto soprattutto ai polonisti per la sua monumentale Historia literatury polskiej (Storia della letteratura polacca) e agli studiosi di storia, di filosofia e di psicologia, per altri suoi importanti lavori ${ }^{2}$. Tuttavia, Wiszniewski fu anche un appassionato italianista (interessato soprattutto alla storia della Penisola italica, ma anche alla cultura, letteratura e costumi italiani e al carattere degli italiani stessi) e, in dettaglio, anche un attento lettore, studioso e traduttore di Dante. Che quest'ultimo campo degli interessi del professore non sia stato mai abbastanza studiato e valutato, è indubbiamente dovuto al fatto che il suo principale contributo alla diffusione del poeta fiorentino in Polonia - il testo delle lezioni universitarie a lui dedicate - rimane ancora inedito, tra altri numerosi scritti dello studioso, presso la Biblioteca Jagellonica di Cracovia. Il presente testo mira a valutare l'apporto di Wiszniewski alla ricezione di Dante in Polonia, sullo sfondo dei suoi generali interessi italianistici e in relazione alle prime pubblicazioni polacche sul Poeta (fino al 1840) e alle tendenze della critica dantesca contemporanea.

Fra i primi bibliografi della fortuna polacca del Poeta (Stanisławski, 1880; Bogusławski, 1885; Grzegorczyk, 1921; Janik, 1921; Koczorowski, 1921; Łempicki, 1929), solo Stanisław Koczorowski e Stanisław Łempicki ricordano il professore cracoviano: uno menziona il suo ma-

1 La rilevanza e l'esigenza di questo tipo di studi è stata sottolineata da molti ricercatori in varie occasioni, per es. Kałuża, 1965, p. 4 e Wasyłenko, 1993, p. 5.

2 Per approfondire si veda: Dybiec, 1970, a cui si deve la gran parte delle informazioni biografiche su Wiszniewski ricordate nel presente testo. 
noscritto intitolato Dante Alighieri (BJ ms. 948 fasc. 7), riportandone la descrizione dal catalogo di Wisłocki, e l'articolo Studia nad Dantem (Studi su Dante) stampato anonimamente nel 1847 sulla rivista Przeglad Poznański, da attribuire - a suo parere - proprio al professore cracoviano (Koczorowski, 1921, pp. 59-60); 1'altro, a sua volta, sebbene elenchi Wiszniewski tra coloro che "si dedicarono agli studi su Dante", in seguito focalizza il discorso, tenuto nel 1929 a Leopoli, sui personaggi di grande fama come Adam Mickiewicz, Juliusz Słowacki ecc. (Łempicki, 1929, p. 3). Più spazio, quasi una pagina e mezzo, dedica a Wiszniewski Mieczysław Brahmer rammentando i molteplici legami tra il professore e l'Italia e le sue lezioni universitarie sul poeta fiorentino. Brahmer cita un breve brano dagli appunti manoscritti dello studioso, menziona anche le sue traduzioni dell'inizio del Convivio e dei due brani della Commedia (l'episodio di Ugolino e quello di Paolo e Francesca), il cui scopo sarebbe integrare e illustrare il discorso sull'Alighieri (Brahmer, 1939, pp. 28-29). Walerian Preisner, autore della bibliografia Dante i jego dzieła $w$ Polsce (Dante e le sue opere in Polonia) colloca Wiszniewski tra i personaggi che nei primi decenni dell'Ottocento (1800-1840) contribuirono di più a diffondere il nome del Poeta in Polonia (Preisner, 1957, pp. 44-47), ma non approfondisce notevolmente il tema degli studi danteschi del professore. Anche lui ascrive a Wiszniewski l'articolo pubblicato nel 1847 su Przeglą Poznański (Preisner, 1957, p. 49). I preziosi studi di Litwornia dal titolo "Dantego któż się odważy tłumaczyć?" Studia o recepcji Dantego w Polsce ("Chi avrà il coraggio di tradurre Dante?" Studi sulla ricezione di Dante in Polonia) non prendono in considerazione la figura dell'autore di Historia literatury polskiej. Visto lo stato della ricerca sulla questione, il contributo dantesco di Wiszniewski richiede ulteriori studi e approfondimenti.

Dalle fonti biografiche risulta che un particolare interesse per la storia e la cultura italiane iniziò a germogliare in Wiszniewski quando egli aveva poco più di vent'anni. Finito il liceo di Krzemieniec, negli anni 1819-1822 il giovane diplomato fece un lungo viaggio all'estero. Al principio si recò in Italia, dove trascorse - tra Venezia, Milano, Padova, Mantova, Bologna e Napoli - circa un anno, ammirando i capolavori d'architettura, d'arte e di letteratura ed esplorando biblioteche. 
Successivamente si spostò in Francia, dove per un anno all'Università di Parigi studiò storia, legge e filosofia. Colto, acuto e laborioso, il giovane Wiszniewski non sfuggì all'attenzione di Adam Czartoryski che stava cercando candidati polacchi per i futuri professori universitari. Nel 1821 il giovane studioso per poco non vinse la cattedra di filosofia dell'Università di Vilnius, alla fine però dovette accontentarsi della borsa che gli permise di continuare per un anno, presso l'Università di Edimburgo, lo studio di filosofia morale e di logica, che - al ritorno in patria - iniziò ad insegnare presso il liceo di Krzemieniec. Nel 1824 Wiszniewski intraprese il suo secondo viaggio in Francia e in Italia, durante il quale visitò, tra l'altro, l'amata Venezia, sulla cui storia possedeva una preziosa collezione di fonti. Rientrato in Polonia, si dedicò per alcuni anni all'agricoltura, agli studi e allo scrivere tra le mura domestiche.

Nel 1831 a Wiszniewski fu conferita la cattedra di storia presso il Dipartimento di Filosofia dell'Università Jagellonica di Cracovia (inizialmente, nel 1830 lo studioso aveva vinto il concorso per la cattedra di filosofia, ma una serie di eventi sfavorevoli lo costrinse ad adeguarsi a una nuova realtà). Nel 1833 gli fu assegnato anche il corso di letteratura. Le sue lezioni ${ }^{3}$ fornivano agli studenti un quadro sintetico della storia della letteratura, non solo europea, ma anche di alcune nazioni extraeuropee, dalle origini fino al 1800, con un'attenzione minore tuttavia per quanto riguarda i secoli XV-XVIII. Il professore esponeva alcuni temi in modo schematico e sommario, per poi soffermarsi invece più a lungo su scrittori e capolavori letterari di grande importanza; era inoltre particolarmente interessato alla nascita e allo sviluppo delle letterature nazionali (Dybiec, 1970, pp. 91-92). I testi delle lezioni e altri materiali manoscritti sulla storia della letteratura, come si è già menzionato, sono custoditi presso la Biblioteca Jagellonica di Cracovia, prima di tutto nel ms. 948 strutturato in 7 fascicoli: 1. Da Alessandro Magno alla morte di Augusto, 2. Storia della letteratura medievale 400-1500, 3. Letteratura antica, 4. Storia della letteratura di varie nazioni, 5. Domande sulla storia della letteratura, 6. Storia della letteratura, 7. Dante Alighieri.

3 Quelle di letteratura, si intende d'ora in poi. Il corso di storia è stato attentamente analizzato e valutato in Dybiec, 1970. 
Il fascicolo 7. del manoscritto 948, composto di 16 fogli e foglietti, contiene gli appunti del professore sulla vita e sull'opera dell'Alighieri, vergati prevalentemente a inchiostro e in piccola parte a matita, databili agli anni Trenta. Si tratta di una stesura provvisoria, con molte correzioni, aggiunte e spostamenti. Il testo, fatta eccezione per lo spazio dedicato ad alcuni brani del poema, ha un carattere sintetico. La biografia di Dante (nella quale l'autore spende qualche parola sulla formazione del Poeta, menziona l'amicizia con Cavalcanti, Giotto e Casella, l'amore per Beatrice, le vicende politiche, l'esilio e l'amicizia con Guido Novello da Polenta) è essenziale: molto più concisa, ma anche più affidabile dal punto di vista storico di quella proposta nel 1817 da Józef Sękowski ${ }^{4}$. Parlando dell'opera dell'Alighieri, il professore si concentra quasi esclusivamente sulla Commedia. Ci introduce all'epoca storica (erano "i tempi di una lotta continua fra il papa e l'imperatore, fra ghibellini e guelfi, i neri e i bianchi; la fine del mondo e il giudizio universale sembravano imminenti") ${ }^{5}$, riassume il contenuto del poema ("nelle immagini dell'Inferno, del Purgatorio e del Paradiso, [il Poeta] collocò tutti i dogmi personificati, tutti i difetti, i vizi e le virtù dei suoi amici e nemici"), ci fornisce le informazioni di base sulla struttura dell'aldilà dantesco e sul ruolo di Virgilio e Beatrice. La Divina Commedia è presentata come il primo componimento (di così alto rango, si intende) scritto in una delle "loquele" sviluppatesi dal latino e Dante come il creatore della lingua italiana ("avendo scoperto un nuovo mondo poetico, scoprì anche una nuova lingua"). Non è solo un luogo comune (ripetuto sia dai neoclassici che dai romantici anche se con toni differenti): Wiszniewski prosegue con una riflessione sulla lingua di Dante, facendo notare che nella Commedia vediamo non poche parole "barbariche" usate in rima, numerosi vocaboli assimilati dal francese, provenzale e latino, molte espressioni nobili ispirate a quelle virgiliane, le quali arricchi-

${ }^{4}$ La pubblicazione di Sękowski, contenente la prima traduzione polacca del III canto dell'Inferno, è la prima abbastanza estesa voce su Dante basata sulle fonti critiche in Polonia. Sękowski fornisce molte informazioni sulla vita di Dante, ma nella sua narrazione i fatti si intrecciano con leggende e aneddoti. Si veda: Sękowski, 1817.

${ }^{5}$ La lezione su Dante, fatta eccezione per alcuni versi del poema citati in italiano, è scritta interamente in polacco. La traduzione italiana dei passi citati è mia. 
scono il volgare italiano, fino a quei tempi usato soprattutto nei contesti quotidiani o per cantare l'amore.

Delle opere minori vengono menzionate il De vulgari eloquentia, il Convivio e il De Monarchia, le quali sono subordinate al discorso sull'opus magnum, il che corrisponde pienamente alla tendenza della critica ottocentesca ${ }^{6}$. La prima dell'elenco, $O$ Wymowie, viene ricordata per spiegare il titolo del poema:

Podzieliwszy w piśmie $O$ wymowie styl na tragiczny, komiczny i elegiacki, Dante, nie znając zapewne wówczas żadnej sztuki dramatycznej, rozumiał, że większa część jego poematu pisana jest stylem środkującym między stylem górnym a elegiackim (siebie między Wergiliuszem i Owidiuszem umieścił), dał poematowi swemu nazwisko Komedii, które dzisiaj nas zadziwia. Późniejsi nazwali tę komedię "boską"

Il Convivio e il De Monarchia, a loro volta, sono citati con lo scopo di dimostrare che nella forma prosastica l'Alighieri non riuscì a sottrarsi al "giogo" della sua formazione scolastica (che, secondo lo studioso, "uccide la mente") e di quella uniformità di ragionamento impostagli dalla logica aristotelica. La voce critica, così evidente, sulla prosa dantesca, è però anche uno spunto per esprimere una grande ammirazione per la sua poesia, nella quale egli, "seguendo l'ispirazione del suo genio, seppe elevarsi così in alto". In realtà già nel Convivio Wiszniewski scorge due volti di Dante: quello scolastico ovvero troppo formale e artificiale e quello poetico - autentico e naturale. Per sostenere la sua tesi cita, nella traduzione propria, due passi dell'opera: il $C v$. I I 1-10 (per

${ }^{6} \mathrm{Ne}$ vediamo un esempio palese in Sękowski che menziona tutte le opere di Dante, ma tratta quelle "minori" brevemente e piuttosto irrispettosamente, per concentrarsi poi esclusivamente sulla Commedia (Sękowski, 1817, p. 480).

7 "Avendo distinto nel De vulgari eloquentia la forma tragica, la forma comica e quella elegiaca, Dante, non essendo probabilmente a conoscenza di nessuna opera drammatica, comprendeva che la maggior parte del suo poema era scritta con lo stile intermedio fra quello superiore e quello elegiaco ([il Poeta] situò se stesso fra Virgilio e Ovidio), diede al suo poema il nome di Commedia, che oggi ci stupisce. I posteriori chiamarono questa commedia «divina»". M. Wiszniewski non fu il primo dei polacchi a spiegare la questione. Lo avevano già fatto Czartoryski, 1774, p. 43, e Sękowski, 1817, pp. 484-485. 
il Dante scolastico) e il Cv. I III 3-5 (per il Dante poetico) ${ }^{8}$. L'Alighieri apprezzato dal professore cracoviano è un poeta che "parla dal cuore". Il ragionamento si avvicina evidentemente all'idea di un Dante romantico, la quale condizionò fortemente la "riscoperta" del Poeta in Europa a cavallo tra il Sette- e l'Ottocento". Wiszniewski illustra il suo discorso con alcuni passi del poema: si sofferma più a lungo sull'episodio di Ugolino e quello di Francesca da Rimini, i quali in parte riassume e in parte traduce in prosa. Va notato che si tratta della seconda traduzione polacca dell'episodio del conte della Gherardesca, preceduta solo dalla "geniale parafrasi" di Mickiewicz ${ }^{10}$, e della prima traduzione polacca di un lungo brano dell'Inf. V. La scelta dei due episodi (caratterizzati come "pieni di bellezza e semplicità, non raffreddati da un'esagerata raffinatezza"), in cui si manifestano "la tenerezza dell'anima e la dolcezza del cuore" del poeta fiorentino conferma il gusto romantico del professore. Wiszniewski sottolinea più volte e fortemente apprezza anche la straordinaria plasticità delle immagini dantesche:

Trudno powiedzieć, co bardziej zadziwiać w tym wielkim obrazie powinno: czy śmiałość pierwszej myśli, czy moc pędzla, który nigdy nie spoczął. Stworzyć zupełnie nowe światy z wyobrażeń, teologiczne [tajemnice] odmalować w żywych i świeżych farbach i oczom ludzkim wystawić (bo każda niemal strofa tego Poematu odrysować się może), świat ten

8 Si tratta della prima traduzione polacca di alcuni passi del trattato filosofico dell'Alighieri, pubblicato interamente in polacco solo nel 2004.

9 Tra i primi a celebrare Dante come padre della poesia moderna e a proclamare un vero e proprio culto dantesco furono i fratelli Schlegel e Schelling (Bartuschat, 2011, pp. 57-64). In Polonia i primi esempi del fenomeno si vedono nei lavori di Kazimierz Brodziński: "podobnie mówić można [że prawie całkiem do literatury romantycznej należą] o dawnych włoskich poetach, szczególniej o Dantym” (Brodziński, 1934, p. 97) e di Maurycy Mochnacki: "Dante, Tasso, Szekspir, Milton, oto są prawodawcy romantycznego parnasu" (Mochnacki, 1828, p. 93). L'idea ebbe il pieno sviluppo negli scritti e nelle opere dei poeti come Mickiewicz, Słowacki, Norwid (si veda, per esempio: Brahmer, 1959; Vincenz, 1980, pp. 174-233; Kuciak, 2003).

${ }^{10} \mathrm{Fu}$ Józef Ignacy Kraszewski a definire in questo modo la traduzione di Mickiewicz pubblicata con il titolo Ugolino. Wyjątek z Boskiej Komedii (e contenente l'Inf. III 1-30 e l'episodio di Ugolino) del 1829 (Kraszewski, 1869, p. 142). 
zaludnić... - [wszystko to] jest oznaką potężnego umysłu natchniętego imaginacją żywą i bujną ${ }^{11}$.

L'immaginazione di Dante (caratterizzata come: "vivace", "ricca", "cupa" ma "ardente" o addirittura "focosa") è un altro tema che affascina lo studioso, il che è indubbiamente correlato alla sua passione per la filosofia della mente umana ${ }^{12}$.

Va notato, tuttavia, che il contributo dantesco del professore cracoviano non è un elogio romantico del Poeta, come ci si potrebbe immaginare vista la sua datazione (anni Trenta dell'Ottocento). Wiszniewski, a differenza ad esempio di Mickiewicz, non si lasciò condizionare dalle alternative: il sentimento o l'intelletto e il classicismo o il romanticismo $^{13}$; le sue convinzioni sull'opera dantesca si devono all'educazione classica e a una ricca cultura filosofica, storica e letteraria. Dante viene presentato come un successore di Omero, dell'Omero vichiano, ovvero come colui che dopo ventidue secoli di stasi diede vita a "una nuova poesia" e il suo poema ci si prospetta come "una fedele e splendida rappresentazione dell'età barbarica che aveva inondato tutta l'Europa, come l'Iliade lo è per i secoli eroici della Grecia"14. Dopo aver rias-

11 "È difficile dire cosa più ci sorprende in questa grande immagine: l'audacia del primo pensiero o la forza del pennello che non si è mai fermato. Creare nuovi mondi dalle immaginazioni, dipingere [i segreti] teologici in colori vividi e freschi e mostrarli agli occhi della gente (perché quasi ogni strofa del Poema può essere disegnata), popolare questi mondi... - [tutto ciò] è un indizio di una mente potente e ispirata da un'immaginazione viva e ricca".

Una simile attenzione per la forza dell'immagine dantesca è fortemente presente nella critica ottocentesca. Si ricordi ad esempio le riflessioni del Leopardi: "ogni parola presso lui è un'immagine", "Dante non solo dipinge da maestro in due colpi [...], ma intaglia e scolpisce dinanzi agli occhi del lettore le proprie idee, concetti, immagini, sentimenti" (cit. da: Vallone, 1958, pp. 67-68).

${ }_{12}$ Nel suo corso di filosofia della mente umana tenuto presso il liceo di Krzemieniec il professore dedicava molto spazio proprio al tema dell'immaginazione: ragionava, tra l'altro, del ruolo dell'immaginazione nelle belle arti, della sua relazione con il genio e con il carattere (Dybiec, 1970, pp. 40-41).

${ }^{13}$ Cf. Vincenz, 1980, pp. 182-184.

14 Il paragone di Dante con Omero, che gode di una lunga tradizione nella critica dantesca, è fortemente presente anche nel dantismo ottocentesco polacco (ne troviamo 
sunto i primi tre canti dell'Inferno (con l'Inf. III 1-3 citato in italiano), Wiszniewski si sofferma sul canto IV, quello degli antichi eroi e dei saggi, dove rimarca la presenza de "il maestro di color che sanno", ovvero Aristotele, e riferisce l'incontro di Dante con i poeti antichi, che in parte traduce e in parte sintetizza ${ }^{15}$, evidenziando in questo modo l'importanza del patrimonio antico per l'opera dantesca. Nell'Alighieri apprezza non solo il sentimento e l'immaginazione, ma anche l'intelletto. Dell'Inferno, oltre agli episodi citati sopra, lo avvince anche il modo in cui il pellegrino riesce ad uscirne, per cui esamina il capovolgimento nel punto centrale della terra:

$\mathrm{W}$ tem to punkcie przestaje działać siła grawitacji, która wszystkie ciała do niego wciąga. Dante, kładąc te słowa w usta Wergiliusza: „Tu passasti il posto al qual si traggon d'ogni parte i pesi”, zadziwia nas tą myślą newtońską ${ }^{16}$.

Nella Commedia il professore, quindi, discerne e apprezza non solo la poesia, ma anche la dottrina. È lontano dal mitizzare o sacralizzare l'Alighieri ${ }^{17}$.

Sullo sfondo di quanto era stato scritto in polacco su Dante prima del 1840, la lezione preparata da Wiszniewski si presenta molto solida-

importanti testimonianze per esempio in: Sękowski, 1817, pp. 481-482; Bończa Tomaszewski, 1822, p. 94; Brodziński, 1872, pp. 221-222; Mickiewicz, 1844, p. 284), dove assume tuttavia diverse sfumature: mentre Sękowski e Mickiewicz riconoscono in Dante e Omero due poeti particolarmente ragguardevoli, ma distanti, Wiszniewski similmente ad esempio al Leopardi (Vallone, 1958, p. 68; Del Gatto, 2017, pp. 59-61) - rileva la continuità del patrimonio).

15 Anche questa traduzione è una grande novità. La prima traduzione dell'Inf. IV che andò in stampa fu quella di J. Krzeczkowski del 1843 (Miszalska, Gurgul, SurmaGawłowska, \& Woźniak, 2007, p. 113).

16 "In questo punto cessa la gravitazione che vi trae tutti i corpi. Dante, facendo affermare a Virgilio: «Tu passasti il posto al qual si traggon d'ogni parte i pesi», ci sorprende con questo pensiero newtoniano".

17 Sul lato opposto c'è invece il lavoro, anteriore di una decina d'anni, di Bończa Tomaszewski, dove Dante si prospetta come un poeta "consacrato dai nostri segreti religiosi" e la sua voce appare così potente da trasformare il mondo intero in poesia (Bończa Tomaszewski, 1822, pp. 94-95). 
mente. In termini di quantità di informazioni fornite essa cede il primato all'articolo di Sękowski del 1817 e non presenta grandi novità di contenuto (a parte le traduzioni, ovviamente, e una breve analisi linguistica del poema $)^{18}$. Tuttavia, il carattere dei due contributi danteschi è del tutto differente, in quanto Sękowski, come si è già accennato, non evita aneddoti e comuni opinioni che costituiscono una parte del culto romantico di Dante. Wiszniewski, sebbene non nasconda le sue convinzioni, è decisamente più fattuale e oggettivo, non perde di vista l'intelletto di Dante e le sue radici antiche. Se si pensa al carattere del dantismo presentato dai principali poeti romantici polacchi, nel quale un ruolo fondamentale fu giocato dalla fede cristiana, dall'impegno politico e dall'esperienza di esilio di Dante, forse ci si rimane stupiti da una quasi assenza di questi temi nel manoscritto dedicato al Poeta. Il fatto può spiegarsi, almeno in parte, con 1'approccio del professore: molto più intellettuale che emotivo.

Il corso di letteratura (similmente a quello di storia) tenuto da Wiszniewski presso il Dipartimento di Filosofia (all'epoca, a Cracovia, un dipartimento di preparazione introduttiva ovvero una tappa necessaria per proseguire poi la formazione presso altri dipartimenti, anche quelli più ambiti: di Medicina e Giurisprudenza) aveva lo scopo di fornire agli studenti una cultura letteraria (e storica) generale. Anche la lezione su Dante è dunque pensata come una scelta delle nozioni di base sulla vita e l'opera del Poeta. È fuori dubbio che lo studioso diede in questo modo un valido apporto all'aumento della conoscenza dell'Alighieri tra i suoi studenti. Una valutazione più accurata dell'importanza del contributo dantesco del professore per la ricezione di Dante in Polonia necessita ulteriori ricerche che oltrepassano gli intenti del presente $\operatorname{articolo}^{19}$.

18 Nella prima metà dell'Ottocento tutti gli articoli su Dante pubblicati in Polonia erano basati sulle idee tratte dalla critica dantesca italiana, francese, tedesca ecc. I veri e propri studi su Dante in Polonia iniziarono più tardi (negli anni Cinquanta dell'Ottocento), con Julian Klaczko, le cui pubblicazioni riscossero un'attenzione internazionale (Preisner, 1957, pp. 50-51, 170-171).

19 Sarebbe interessante esaminare attentamente l'attività successiva degli ex studenti di M. Wiszniewski, tra i quali risalta subito per esempio Gustaw Ehrenberg, autore della prima traduzione pubblicata della Vita nuova (1880). Un breve elenco dei più 
Wiszniewski partecipò alla diffusione di Dante (e della cultura italiana in generale) in Polonia anche tramite il suo libro Podróż do Włoch, Sycylii i Malty (Viaggio in Italia, in Sicilia e a Malta) pubblicato nel 1848. Si tratta dei ricordi del terzo viaggio del professore nella patria di Dante, compiuto nel 1845, ufficialmente con lo scopo di dare sollievo al corpo e alla mente, dopo anni di faticosi impegni scientifici e didattici, ma probabilmente anche con l'intenzione di svolgere alcune ricerche per la sua Historia literatury polskiej nelle biblioteche italiane. Il professore visitò, tra l'altro, Venezia, Padova, Vicenza, Verona, Milano, Pavia, Genova, Pisa, Napoli, Sorrento, Malta, Sicilia, Firenze, Bologna e Ferrara. Nel suo libro i ricordi di viaggio si intrecciano con delle digressioni e leggende, la narrazione è vivida e plastica, intessuta di numerose citazioni letterarie (dai classici greci e romani, per Dante, Petrarca, Boccaccio, Tasso, Alfieri, Foscolo, Manzoni, Byron, Goethe e altri, fino alla più recente letteratura americana) usate con molta leggerezza e spontaneità ${ }^{20}$. Dante viene menzionato con frequenza in varie occasioni e in vari contesti. A Venezia, per esempio, Wiszniewski si serve delle terzine dantesche, citate in italiano (Inf. XXI 7-15), per descrivere l'Arzanà. Parlando di Verona cita l'Epistola a Cangrande della Scala, traducendone alcuni passi dal latino, ricorda l'esilio del poeta fiorentino preannunciatogli da Cacciaguida, e la leggendaria inimicizia dei Cappelletti e dei Montecchi, menzionata già nella Commedia. Descrivendo il soggiorno a Pisa, richiama abilmente alla memoria del lettore il famoso episodio infernale:

Potem przejeżdżaliśmy około wieży Ugolina, teraz kamienicy, w której oprócz pięknej Włoszki z niej wyglądającej nic szczególnego nie widzieliśmy i pewnie byśmy nie szukali, gdyby nie czarujące pióro Dantego. (Wiszniewski, 1982, p. 223) ${ }^{21}$

noti ex studenti del professore si ha in Dybiec, 1970, p. 94. Le liste complete sono da recuperare dall'archivio universitario.

20 Per una caratteristica più dettagliata dello stile di Podróż si veda la postfazione di Henryk Barycz in: Wiszniewski, 1982, pp. 518-526.

21 "Dopo siamo passati davanti alla torre di Ugolino, oggi un palazzo, in cui, a parte una bella ragazza italiana alla finestra, non abbiamo notato niente di speciale e probabilmente non l'avremmo neanche cercato se non fosse per l'affascinante penna di 
In un altro luogo, raccontando di Monte Cassino, l'autore riporta alcuni versi della Commedia ( $P$ d. XXII 37-39), ma non in italiano come era solito fare, ma nella propria traduzione:

Wierzchołek wzgórza, gdzie u stromej ściany

Przylgnęło drobne murami Cassino,

Lud zamieszkiwał krnąbrny a zbłąkany. (Wiszniewski, 1982, p. 423)

Podróż di Wiszniewski fu un testo di rilievo nella precedente e contemporanea letteratura polacca sull'Italia e fonte d'ispirazione per altri viaggiatori ottocenteschi che scoprivano l'Italia con il libro del professore in mano (Dybiec, 1970, pp. 252-254). Anche le citazioni dantesche, dunque, ebbero molti lettori.

Come si è accennato sopra, da alcuni studiosi Wiszniewski è considerato anche l'autore dell'articolo Studia nad Dantem del 1847, strutturato in due sezioni: I. Zagajenie rzeczy (Nota introduttiva) e II. Wstęp historyczny (Introduzione storica). Il testo tratta in modo molto diffuso dell'Italia medievale e contiene scarse informazioni sull'Alighieri, su cui l'autore prometteva di soffermarsi nelle successive puntate; tuttavia, il seguito non venne mai pubblicato. L'attribuzione dello studio è incerta e problematica: Koczorowski e Preisner lo ascrivono al professore cracoviano, ma non ne forniscono né prove né indizi concreti (il primo afferma soltanto: "a giudicare dallo stile e dal metodo - un lavoro di Wiszniewski" (Koczorowski, 1921, pp. 59-60), il secondo, pur non avendo indagato la questione, la complica ulteriormente, ponendo, nella sua bibliografia, Studia nad Dantem sia accanto al nome di Wiszniewski, sia accanto al nome di Antoni Celiński) ${ }^{22}$. Bogusławski era invece convinto che gli studi in oggetto, definiti da lui "la prima seria manifestazio-

Dante". Il passo può fungere da esempio della leggerezza con cui Wiszniewski intreccia i propri ricordi con delle citazioni e allusioni letterarie. In quel caso, nient'altro deve essere aggiunto: negli anni Quaranta l'episodio di Ugolino è ormai ben noto agli intellettuali polacchi.

${ }^{22}$ Preisner, 1957: p. 49: il testo in questione è ascritto, senza nessuna motivazione, a Wiszniewski; p. 155: il testo è assegnato a Ludwik Antoni Celiński (con una fuorviante nota sul contenuto degli studi di Celiński, della quale evidentemente si servì poi Litwornia raccogliendo informazioni su quel traduttore di Dante - cf. Litwornia, 2005, 
ne nostrana su Dante", furono scritti da Celiński ${ }^{23}$. Anche in Litwornia il testo viene citato nella sezione dedicata a Celiński, ma è evidente che l'autore di "Dantego któż się odważy ttumaczyć?" ha tenuto conto di quanto su Celiński si legge in Preisner.

A causa della perdita, durante la Seconda guerra mondiale, di una consistente corrispondenza di Wiszniewski, risulta impossibile ricostruire pienamente l'apporto del professore ai periodici polacchi dell'epoca. Pare tuttavia che esso fu limitato, in quanto per lo studioso, concentrato principalmente su un intenso lavoro di ricerca e sulla redazione dei suoi libri, la collaborazione con delle riviste era un'attività assolutamente aggiuntiva. Dato che Wiszniewski non aveva l'abitudine di firmare i suoi contributi ai periodici, nelle sue biografie si notano delle notevoli divergenze su quanti e quali testi fossero effettivamente prodotti dal professore (Dybiec, 1970, pp. 132-133). Incontestabile, pur modesta, risulta la sua cooperazione con alcune testate edite in Wielkopolska, come Tygodnik Literacki, Orędownik e Przyjaciel Ludu. Quanto a Studia nad Dantem, tra gli indizi che possono suggerire, ma certamente non comprovare, un'eventuale paternità di Wiszniewski ci sono: l'argomento storico che pur dovendo fungere solo da introduzione è esposto in modo molto esteso (forse questo intendeva, per "stile e metodo" del professore, Koczorowski?) e la mancanza della continuazione: in effetti, lo studioso cracoviano spesse volte si entusiasmava per nuovi progetti scientifici, per poi abbandonarli (Dybiec, 1970, pp. 328-329). Eppure, il carattere dell'articolo in questione (in particolare una forte enfasi sul ruolo edificante della Provvidenza, della dignità papale e della devozione cristiana nella storia, nonché la glorificazione del poeta fiorentino, denominato sempre con la forma "Dant", assente nel manoscritto 94824) ci porta inevitabil-

p. 182); p. 210: il testo è nuovamente assegnato a Wiszniewski, stavolta con il punto interrogativo.

23 Bogusławski ricorda inoltre che gli studi sarebbero stati menzionati da Kraszewski e ne fornisce una breve analisi e critica (Bogusławski, 1885, pp. 632-634). In realtà in Kraszewski troviamo solo una menzione del nome di Celiński, senza nessuna precisazione di quali studi egli fosse autore (Kraszewski, 1869, p. 189).

24 La forma "Dant", ispirata al francese, oggi antiquata, era abbastanza diffusa nel polacco ottocentesco. 
mente a diffidare dell'attribuzione di Studia nad Dantem a Wiszniewski. Va tenuto in considerazione che il testo può essere anche, almeno in parte, una traduzione o un rifacimento, forse degli studi di Antoine-Frédéric Ozanam, Claude Charles Fauriel, Ludwig Gottfried Blanc (suggeriti da Kraszewski ${ }^{25}$ ) o di altri critici stranieri, il che complica ulteriormente il problema dell'identificazione del suo autore. Le ricerche che vanno eseguite a tale scopo oltrepassano l'interesse del presente articolo. Vista l'incerta attribuzione del testo, si rinuncia quindi a una sua analisi nel contesto dell'opera dello studioso.

Come si è visto, le inclinazioni italianistiche di Michał Wiszniewski, che nel 1849 rinunciò alla cattedra e si trasferì in Italia, sono più che forti. Il suo apporto alla ricezione di Dante in Polonia consisté soprattutto nel diffondere e divulgare la vita e l'opera del Poeta tra gli studenti del corso di letteratura tenuto per molti anni presso 1'Università Jagellonica di Cracovia e tra i lettori del libro Podróż do Włoch, Sycylii i Malty. Nel suo approccio all'opera del poeta toscano si scorgono sia delle tracce del gusto romantico che quelle di tendenza neoclassica. Il professore apprezza nella Commedia sia la poesia che la dottrina. Una grande novità proposta da Wiszniewski sono le traduzioni di un lungo passo del Convivio e dei tre episodi dell'Inferno, che meritano uno studio a parte. Si è visto che un ulteriore approfondimento è indispensabile anche per quanto riguarda l'attribuzione di Studia nad Dantem. Il contributo dantesco del professor Wiszniewski continua ad essere un interessante campo da indagare.

25 Si veda la n. 23. Anche Bogusławski scorge in Studia nad Dantem delle affinità agli studi di Ozanam (Bogusławski, 1885, p. 633). Nel 1845, tra le notizie di attualità, su Przegląd Poznański apparve una nota anonima con l'informazione che "Ozanam, un eccellente professore della Sorbona di Parigi e autore dell'opera Dante e la filosofia [cattolica nel tredicesimo secolo], stava pubblicando sul periodico Le Correspondant una serie di articoli sulle fonti poetiche della Divina Commedia" (Przegląd Poznański, 1845 , p. 106). Il fatto rende ancora più necessaria la verifica di quanto l'articolo del 1847 sia effettivamente vicino agli studi del critico francese. In margine va chiarito anche che in Litwornia la nota viene erroneamente presentata come un articolo di Celiński dedicato alle fonti del poema (Litwornia, 2005, p. 182). 


\section{BIBLIOGRAFIA}

Michał Wiszniewski, Dante Alighieri, Biblioteca Jagellonica di Cracovia, Ms. 948, fasc. 7.

Bartuschat, J. (2011). Dante in Germania in età romantica. In M. Ciccuto (Ed.), Letture Classensi, vol. 39: Letture e lettori di Dante. L'età moderna e contemporanea (pp. 47-70). Ravenna: Angelo Longo Editore.

Bogusławski, K. (1885). Dante w Polsce. Niwa, 14, 625-635, 746-770.

Bończa Tomaszewski, D. (1822). Pisma wierszem $i$ proza oryginalne i ttumaczone (vol. 2). Warszawa: N. Glücksberg.

Brahmer, M. (1939). Z dziejów włosko-polskich stosunków kulturalnych. Studia $i$ materiały. Warszawa: Nakładem Towarzystwa Literackiego im. A. Mickiewicza.

Brahmer, M. (1959). Dante, le grand émigré, et le romantisme polonais. In W.P. Friederich (Ed.), Comparative Literature: Proceedings of the Second Congress of the ICLA at the University of North Carolina, September 8-12, 1958 (vol. 2). Chapel Hill (N.C.): University of North Carolina Press.

Brodziński, K. (1872). Pisma (vol. 2). Poznań: Drukiem J.I. Kraszewskiego. Brodziński, K. (1934). Pisma estetyczno-krytyczne (vol. 1). Warszawa: Wydawnictw Gabinetu Filologicznego Towarzystwa Naukowego Warszawskiego.

Czartoryski, A.K. (1774). Panna na wydaniu: Komedia we dwóch aktach (2nd ed.). Warszawa: Michał Gröll.

Del Gatto, A. (2017). "Una lunga lirica": La Divina Commedia di Leopardi. In J. Szymanowska, \& I. Napiórkowska (Eds.), Il Dante dei moderni. La Commedia dall'Ottocento a oggi (pp. 59-69). Vicchio: Lo Gisma.

Dybiec, J. (1970). Michat Wiszniewski. Życie i twórczość. Wrocław-Warszawa-Kraków: Wydawnictwo Polskiej Akademii Nauk.

Janik, M. (1921). Dante w Polsce. Nowa Reforma, 40 (217), 1.

Kałuża, Z. (1965). Najnowsze polskie prace o Dantem. Zeszyty naukowe KUL, $8(4), 47-52$.

Koczorowski, S.P. (1921). Dante w Polsce. Bibliografia przekładów dziet jego tudzież prac jemu poświęconych w Polsce lub przez Polaków wykonanych ze wstępem, przypisami i wzorami przekładów. Kraków: Polska Akademia Umiejętności. 
Kraszewski, J.I. (1869). Dante: studia nad Komedia Boska. Roczniki Towarzystwa Przyjaciót Nauk Poznańskiego, 5, 95-189.

Kuciak, A. (2003). Dante romantyków. Recepcja Boskiej Komedii u Mickiewicza, Słowackiego, Krasińskiego i Norwida. Poznań: Wydawnictwo Naukowe UAM.

Litwornia, A. (2005). „Dantego któż się odważy tlumaczyć?” Studia o recepcji Dantego w Polsce. Warszawa: Instytut Badań Literackich PAN.

Łempicki, S. (1929). Dante a Polska. Gazeta Lwowska, 268, 3-4, 269, 3-4, 270, 3-4, 271, 3-4.

Mickiewicz, A. (1844). Pisma Adama Mickiewicza (na nowo przejrzane, dopetnione i za zezwoleniem jego...). Paryż: Bourgogne et Martinet.

Miszalska, J., Gurgul, M., Surma-Gawłowska, M., \& Woźniak, M. (2007). Od Dantego do Fo. Włoska poezja i dramat w Polsce (od XVI do XXI wieku). Kraków: Collegium Columbinum.

Mochnacki, M. (1828). [senza titolo]. Gazeta Polska, 24, 93-95.

Preisner, W. (1957). Dante i jego dzieła w Polsce. Bibliografia krytyczna $z$ historycznym wstepem / Dante e le sue opere in Polonia. Bibliografia critica con una introduzione storica. Torun: Towarzystwo Naukowe w Toruniu.

Przeglad Poznański (1845). [Boska Komedia Dantego...], 1, 106.

Przegląd Poznański (1847). Studia nad Dantem, 4, 313-384.

Sękowski, J. (1817). Z „Boskiej Komedii” Danta Alighieri o Piekle pieśń

III, z poprzednią wiadomością o życiu i pismach tego autora. Dziennik Wileński, 6, 473-492.

Stanisławski, A. (1880). Dante w Polsce. Gazeta Lwowska, 70, 4.

Vallone, A. (1958). La critica dantesca nell'Ottocento. Firenze: Olschki.

Vincenz, S. (1980). Z perspektywy podróży. Kraków: Znak.

Wasyłenko, W. (1993). Polskie losy Dantego w wieku XIX. Prolegomena do „,zaginionego” ttumaczenia „Boskiej Komedii” dokonanego przez J.I. Kraszewskiego. Poznań: Wydawnictwo Poznańskiego Towarzystwa Przyjaciół Nauk.

Wiszniewski, M. (1982). Podróż do Włoch, Sycylii i Malty. Warszawa: Państwowy Instytut Wydawniczy.

Riassunto: Lo studio si concentra sull'interesse per Dante dimostrato da Michał Wiszniewski, professore di storia e letteratura presso l'Università Jagellonica di Cracovia, e un appassionato italianista. L'obiettivo che ci si pone è quello di delineare il contributo di Wiszniewski per la ricezione polacca di Dante nei primi decenni del XIX secolo. Nello studio vengono ricordati i tre viaggi italiani del professore e il suo libro Viaggio in Italia, in Sicilia e a Malta contenente numerose 
menzioni dell'Alighieri. La parte principale dello studio è dedicata all'analisi del suo manoscritto Dante Alighieri, custodito presso la Biblioteca Jagellonica (ms. 948 fasc. 7), finora non esaminato attentamente. Si tratta di un abbozzo di una parte del corso di letteratura tenuto da Wiszniewski negli anni Trenta. La lezione riguarda la vita e l'opera di Dante (in particolare la Commedia). Il professore distingue due volti di Dante: quello scolastico (in prosa) e quello poetico (in versi), spiega il titolo del poema e la struttura dell'aldilà, ammira la straordinaria plasticità delle immagini dantesche, traduce in polacco un passo del Convivio e tre episodi infernali. Il contenuto del discorso viene confrontato con i precedenti e contemporanei studi polacchi, di numero assai limitato, sul Poeta, nonché con le tendenze generali della critica dantesca dell'epoca e con il dantismo dei poeti romantici polacchi. L'analisi eseguita dimostra che il contributo dantesco di Wiszniewski è in linea di massima vicino alla critica romantica, ma contiene anche dei punti di tendenza neoclassica. Nel presente studio non si prende in considerazione l'articolo Studia nad Dantem del 1847, in quanto si tratta di un testo di incerta attribuzione, come si prova a dimostrare. È perciò evidente che il problema dell'apporto di Wiszniewski alla ricezione di Dante in Polonia resta ancora un interessante campo da indagare.

Parole chiave: Wiszniewski, Dante, Polonia, ricezione, Ottocento 